\title{
Diagnostic tardif de la syphilis maternelle et congénitale : une épidémie mal reconnue?
}

\author{
Zachary Dionisopoulos' ${ }^{1}$ Fatima Kakkar², Ana C Blanchard²*
}

\section{Résumé}

La syphilis est une infection causée par les spirochètes de Treponema pallidum. Le diagnostic de cette maladie transmise sexuellement peut être manqué, en partie en raison de la nature indolore des ulcères génitaux au stade primaire. Au Canada, les femmes se soumettent à un dépistage de la syphilis au premier trimestre de grossesse, mais à ce jour, le dépistage en fin de grossesse n'est pas effectué dans toutes les provinces; par conséquent, il peut se produire une transmission verticale non détectée de la syphilis. Cette situation souligne l'importance de reconnaître la syphilis congénitale chez les nourrissons et les jeunes enfants qui ont des problèmes de croissance inexpliqués et des anomalies biochimiques et hématologiques. La syphilis congénitale demeure un diagnostic rare, mais dans le contexte de l'augmentation des taux de syphilis au Canada au cours des dernières années, les cliniciens devraient tenir compte de ce diagnostic chez les nourrissons présentant des manifestations cliniques compatibles.

Citation proposée : Dionisopoulos Z, Kakkar F, Blanchard AC. Diagnostic tardif de la syphilis maternelle et congénitale : une épidémie mal reconnue? Relevé des maladies transmissibles au Canada 2022;48(2/3):130-3. https://doi.org/10.14745/ccdr.v48i23a10f

Mots-clés : syphilis, infections transmissibles sexuellement, grossesse, infection congénitale, épidémiologie
Cette oeuvre est mise à la disposition selon les termes de la licence internationale Creative Commons Attribution 4.0

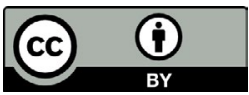

\begin{abstract}
Affiliations
${ }^{1}$ Programme de résidence en pédiatrie, Département de pédiatrie, CHU Sainte-Justine, Université de Montréal, Montréal, QC

2 Service des maladies infectieuses, Département de pédiatrie, CHU Sainte-Justine, Université de Montréal, Montréal, QC
\end{abstract}

\section{${ }^{\star}$ Correspondance :}

ana.blanchard@umontreal.ca

\section{Introduction}

La syphilis congénitale peut être difficile à diagnostiquer, car les symptômes peuvent être semblables à d'autres conditions. De plus, les cliniciens ne tiennent pas toujours compte de la syphilis congénitale en raison de la rareté de ce diagnostic et du dépistage de la syphilis chez les femmes au cours du premier trimestre de la grossesse. Nous présentons ici le cas d'un nourrisson atteint d'un retard de croissance intra-utérine (RCIU) et d'une infection congénitale connue à cytomégalovirus (CMV) qui présentait une anémie persistante, une thrombocytopénie et une élévation des enzymes hépatiques. Ce cas met en évidence la nécessité d'un indice élevé de suspicion chez les nourrissons qui présentent ces constatations et soulève d'importantes questions au sujet de la nécessité du dépistage au troisième trimestre chez les femmes enceintes au Canada.

\section{Cas}

Un nourrisson de deux mois a été admis à l'hôpital pour une évaluation de bicytopénie et hépatite en septembre 2021. II est né d'une mère G1P1 de 21 ans. Au cours du premier trimestre, elle a eu un résultat séronégatif pour le $\mathrm{VIH}$, I'hépatite $B$, I'hépatite $C$ et la syphilis. Elle était immunisée contre la rubéole et les résultats des tests d'urine étaient négatifs pour la chlamydia et la gonorrhée. La grossesse a été compliquée par un oligohydramnios et $\mathrm{RCIU}$ au troisième trimestre. Le bébé est né prématurément à 35 semaines $1 / 7$ de gestation par accouchement vaginal, avec comme score d'Apgar 9-10-10. Les paramètres de naissance ont confirmé le RCIU avec un poids à la naissance de 1,910 kg (score $z-1,48$, centile 6,97), une longueur de $46 \mathrm{~cm}$ (score $z-0,09$, centile 46,47 ) et une circonférence crânienne de $31 \mathrm{~cm}$ (score z -0,86, centile 19,53). Dans le cadre de son examen de RCIU, des tests de réaction en chaîne par polymérase (PCR) par salive et urine pour le CMV ont été effectués au jour zéro et au jour cinq de vie et ont révélé des résultats positifs à 33884416 et à 3801894 copies/ml, respectivement. Le nourrisson avait alors une formule sanguine complète ainsi que des enzymes hépatiques normales. Une échographie transfontanellaire a révélé la présence de petits kystes parenchymateux colloïdaux dans le noyau caudé droit d'origine inconnue, sans calcifications intracrâniennes. L'imagerie par résonance magnétique cérébrale était normale. Les examens ophtalmologique et audiologique étaient normaux. II a reçu du valganciclovir à une dose de $16 \mathrm{mg} / \mathrm{kg} /$ dose deux fois par jour, avec un plan de traitement de six mois. Il a eu congé à la maison à 38 semaines et $4 / 7$.

Lors d'un rendez-vous de suivi à l'âge de six semaines, on a constaté que le nourrisson avait un taux d'hémoglobine de $66 \mathrm{~g} / \mathrm{l}$, des plaquettes à $77 \times 10^{9} / \mathrm{l}$ et des leucocytes de $10 \times 10^{\circ} / \mathrm{L}$. Son taux d'alanine aminotransférase était de $253 \mathrm{U} / \mathrm{l}$, I'aspartate aminotransférase de $254 \mathrm{U} / \mathrm{l}$, le gamma-glutamyl transférase de 
$256 \mathrm{U} / \mathrm{I}$, la phosphatase alcaline de $690 \mathrm{U} / \mathrm{l}$, I'albumine de $29 \mathrm{~g} / \mathrm{l}$ et son coagulogramme était normal. Son équipe de soins a cru que l'anémie et les anomalies des enzymes hépatiques étaient dues à une toxicité du valganciclovir, de sorte que le traitement a été mis en suspens. Il a été revu en clinique une semaine plus tard sans amélioration des résultats de ses analyses sanguines; par conséquent, il a été hospitalisé dans le service de pédiatrie pour des investigations plus poussées.

À son admission, il était afébrile et son état général était bon. À l'examen physique, il avait une hépatosplénomégalie et une desquamation diffuse légère. II était irritable, mais consolable. Un examen approfondi a été effectué, incluant des cultures de sang et d'urine, et des PCR viraux sanguins pour les virus Epstein-Barr, parvovirus B19, adénovirus, herpès simplex et CMV. Tous les résultats étaient négatifs. II a dû recevoir une transfusion de globules rouges, car son taux d'hémoglobine était tombé à $57 \mathrm{~g} / \mathrm{l}$.

Après 10 jours à l'hôpital, le nourrisson a développé une fièvre insidieuse. Compte tenu de l'anémie persistante et inexpliquée, l'équipe commençait à considérer une ponction de moelle osseuse. En posant des questions à la mère alors qu'elle était seule, elle a décrit des premiers symptômes grippaux après l'accouchement, ainsi que des lésions génitales. De plus, l'équipe a constaté une éruption papuleuse palmaire bilatérale érythémateuse (figure 1), apparue au cours des trois semaines précédentes. L'équipe a immédiatement demandé une sérologie de syphilis au patient et à sa mère. Ils avaient tous deux des dosages immunoenzymatiques fortement positifs pour la syphilis et une sérologie réaginique de 1:64. Une ponction lombaire a été effectuée sur le bébé, et l'analyse du liquide céphalorachidien a révélé un décompte cellulaire, glucose, protéines, ainsi qu'un test du Laboratoire de recherche sur les maladies vénériennes tous normaux. L'équipe a procédé à une imagerie par résonance magnétique cérébrale et le résultat était normal. Une étude du squelette n'a pas été concluante pour la syphilis congénitale, mais des réactions périostées légèrement augmentées ont été observées le long des fémurs et du tibia droit (figure 2). L'examen placentaire n'a révélé qu'une seule zone de villosité, et aucun spirochète n'a été observé sur les tests immunochimiques.

Le nourrisson a été traité avec un traitement intraveineux de pénicilline $\mathrm{G}$ pendant 10 jours, et sa fièvre et ses anomalies sanguines se sont résolues rapidement. L'anticorps réaginique répété à la fin du traitement était de 1:32. Le patient a reçu son congé et a fait l'objet d'un suivi à la clinique d'infection congénitale. Sa mère a été référée vers un centre de maladies infectieuses pour adultes, où elle a reçu un traitement approprié et un suivi sérologique.
Figure 1 : Éruption cutanée, femme atteinte de syphilis au stade secondaire, Montréal, 2021

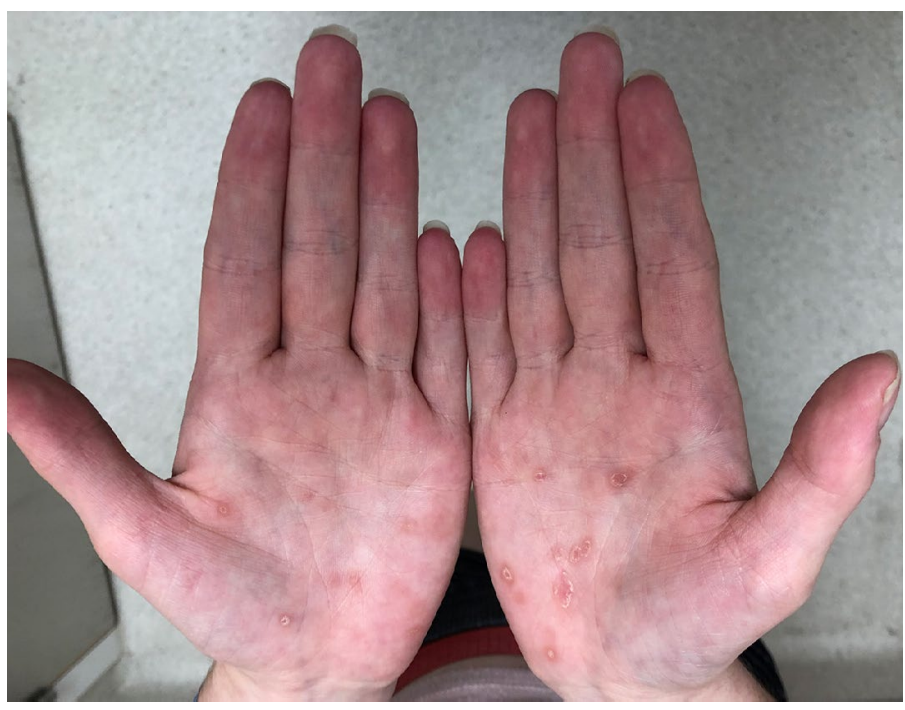

Figure 2 : Radiographie du fémura droit d'un nourrisson atteint de syphilis congénitale, Montréal, 2021

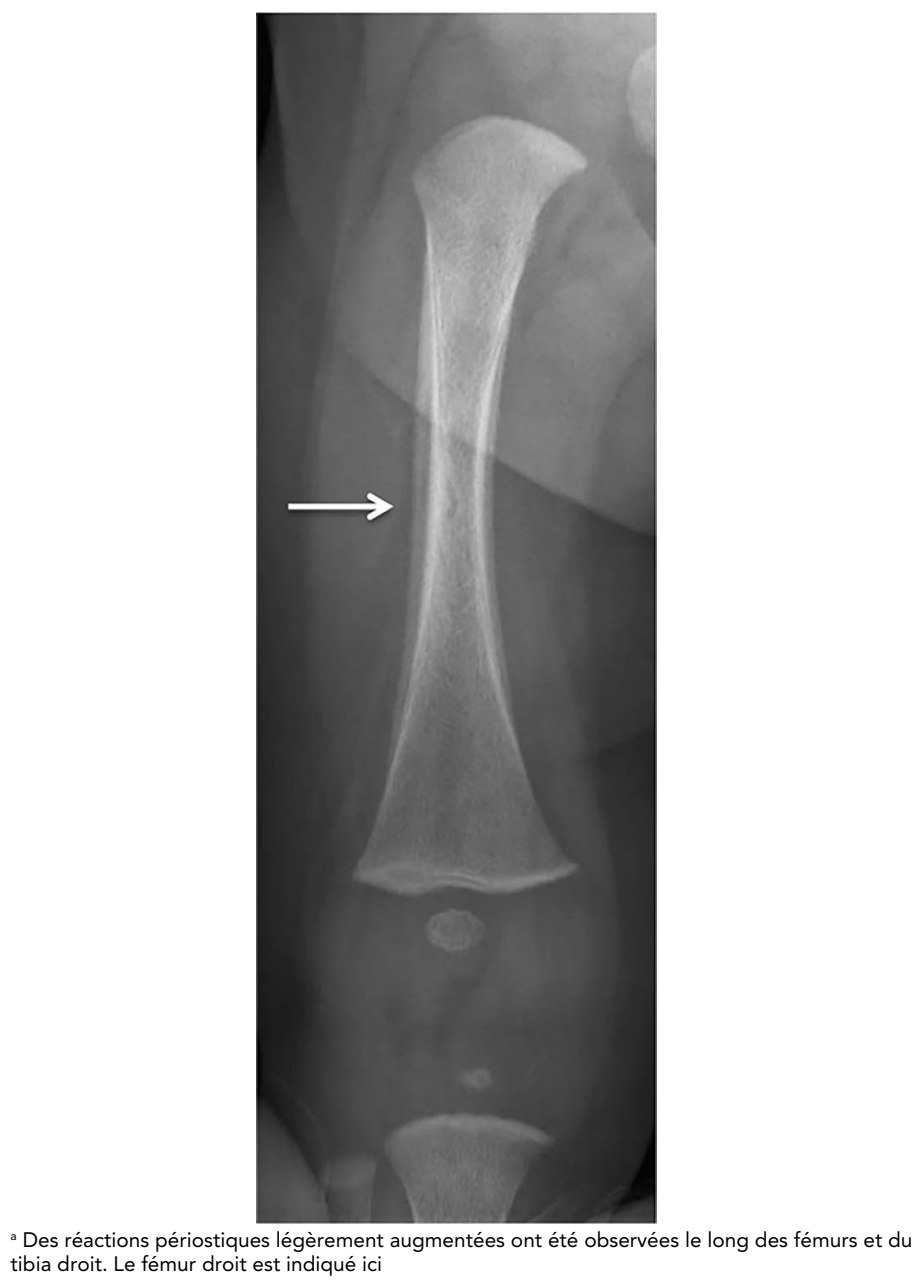




\section{Discussion}

Le taux de syphilis au Canada a augmenté de 85,6 \% de 2010 à 2015, passant de 5,0 à 9,3 cas pour 100000 habitants. Même si ce taux était plus élevé chez les hommes que chez les femmes et qu'on pensait qu'il était en grande partie attribuable aux hommes qui ont des rapports sexuels avec des hommes, on a observé une augmentation de $27,8 \%$ chez les femmes, avec les personnes âgées de 20 à 39 ans affichent les taux les plus élevés (1). Dans un rapport récent, les taux nationaux de syphilis ont augmenté de $124 \%$ entre 2016 et 2020 , alors que la plus forte augmentation a été observée chez les femmes (740 \%). Près du tiers des cas se sont produits chez des femmes (contre seulement $8 \%$ en 2016) (2). De plus, l'incidence de la syphilis congénitale a augmenté au cours des dernières années au Canada, passant de 2,5 cas pour 100000 femmes âgées de 15 à 39 ans en 2016 à 39,7 cas pour 100000 femmes âgées de 15 à 39 ans en 2020 (2). Cette hausse alarmante de la syphilis chez les femmes Canadiennes et leurs nourrissons demande une attention urgente, comme on l'a déjà signalé récemment aux États-Unis et en Australie $(3,4)$. Le Programme canadien de surveillance pédiatrique a récemment entrepris une étude transversale dans l'ensemble du Canada pour décrire l'épidémiologie de la syphilis congénitale, déterminer les facteurs de risque chez les mères d'enfants touchés et décrire le processus d'enquête et de prise en charge de ces enfants (5).

La syphilis congénitale se produit par transmission verticale, pendant la grossesse et l'accouchement. Elle peut entraîner des conséquences graves pour le nourrisson, notamment une perte auditive sensorielle, des troubles neurodéveloppementaux, des déformations musculo-squelettiques et la mort (6). Le risque de transmission varie à plus de $80 \%$ selon le stade de la maladie chez la mère, et le risque de transmission est plus élevé lorsque la maladie est au stade primaire ou secondaire (6). Avec un traitement approprié pendant la grossesse, le risque de syphilis congénitale diminue à $1 \%$ à $2 \%$ (7). Bien que la syphilis fasse régulièrement l'objet de tests au cours du premier trimestre de la grossesse, les tests sérologiques chez les femmes enceintes plus tard au cours de la grossesse ne sont actuellement pas une pratique répandue au Canada. Ces tests se font habituellement de façon ciblée dans les milieux à haut risque, mais les définitions de ces milieux varient. Le dépistage au troisième trimestre a été mis en place en 2019 en Colombie-Britannique en raison de deux cas graves de syphilis congénitale (8).

Les nourrissons peuvent être complètement asymptomatiques à la naissance (9); cependant, un large éventail de manifestations cliniques peut se produire dans le contexte d'une infection congénitale, notamment la prématurité, la petite taille pour l'âge gestationnel, l'anémie, la thrombocytopénie, I'hépatosplénomégalie et l'ostéite (9). Les pédiatres et les médecins de famille doivent être au courant de ces manifestations cliniques, soupçonner rapidement la possibilité de syphilis congénitale, obtenir des examens et mettre en place une prise en charge rapide pour prévenir les complications liées au diagnostic tardif. Un historique détaillé des symptômes et des facteurs de risque épidémiologiques devrait être obtenu auprès des mères en toute confidentialité, comme l'illustre ce cas, afin de pouvoir poser un diagnostic en temps utile. Tous les enfants qui présentent des évidences cliniques ou sérologiques de syphilis congénitale doivent être traités par pénicilline $G$ pendant 10 à 14 jours, selon la gravité de leur maladie. Tous les enfants potentiellement exposés à la syphilis in utero doivent faire l'objet d'un suivi clinique et sérologique (9).

Il convient de noter que la coinfection avec le CMV et la syphilis chez les nouveau-nés demeure largement absente de la littérature. Comme le CMV est de plus en plus reconnu comme une cause principale d'infection congénitale, il peut être détecté plus fréquemment; par conséquent, il peut y avoir plus de descriptions de cas de coinfection à l'avenir. La survenue de ces coinfections et leurs impacts sur les issues cliniques à long terme des nourrissons affectés demandent des études plus poussées.

\section{Conclusion}

Ce cas souligne l'importance de considérer la syphilis congénitale chez les nourrissons présentant une hépatosplénomégalie et des anomalies hématologiques et biochimiques inexpliquées, malgré un dépistage négatif au cours du premier trimestre de la grossesse. Les initiatives de surveillance, comme celles dirigées par le Programme canadien de surveillance pédiatrique, sont utiles pour décrire l'épidémiologie et la gravité de la syphilis congénitale qui est potentiellement sous-diagnostiquée chez les enfants Canadiens. Avec la résurgence actuelle de la syphilis au Canada, les autorités de santé publique des différentes provinces et territoires devraient envisager des tests de dépistage au troisième trimestre de grossesse chez les femmes enceintes.

\section{Déclaration des auteurs}

Tous les auteurs ont participé à la prise en charge du patient: Z. D. et A. C. B. ont posé le diagnostic et assuré la prise en charge initiale et les conseils à la famille; F. K. a assuré le suivi clinique et la prise en charge à long terme. Z. D. a rédigé la version préliminaire de l'article. A. C. B. a supervisé la préparation et la révision de l'article. Tous les auteurs ont lu et approuvé l'article final.

Le contenu et le point de vue exprimés dans cet article sont ceux des auteurs et ne reflètent pas nécessairement ceux du gouvernement du Canada.

\section{Intérêts concurrents}

Aucun. 


\section{Remerciements}

Les auteurs aimeraient remercier la mère du patient, qui a accepté que ces données soient transmises à la communauté scientifique afin de sensibiliser la population à la syphilis.

\section{Financement}

Ces travaux n'ont pas reçu de subvention particulière d'organismes de financement des secteurs public, commercial ou sans but lucratif.

\section{Références}

1. Choudhri Y, Miller J, Sandhu J, Leon A, Aho J. La syphilis infectieuse et la syphilis congénitale au Canada, de 2010 à 2015. Relevé des maladies transmissibles au Canada. 2018;44(2):47-53. DOI

2. Agence de la santé publique du Canada. Syphilis infectieuse et syphilis congénitale au Canada, 2020 (infographie). Ottawa (ON) : ASPC; (modifié 2021). https://www.canada. $\mathrm{ca} / \mathrm{fr} /$ sante-publique/services/publications/maladieset-affections/syphilis-infectieuse-syphilis-congenitalecanada-2020.html
3. Gilmour LS, Best EJ, Duncanson MJ, Wheeler BJ, Sherwood J, Thirkell CE, Walls T. High Incidence of Congenital Syphilis in New Zealand: A New Zealand Pediatric Surveillance Unit Study. Pediatr Infect Dis J 2022;41(1):66-71. DOl PubMed

4. Kimball A, Torrone E, Miele K, Bachmann L, Thorpe P, Weinstock H, Bowen V. Missed Opportunities for Prevention of Congenital Syphilis - United States, 2018. MMWR Morb Mortal Wkly Rep 2020;69(22):661-5. DOI PubMed

5. Canadian Paediatric Surveillance Program. Protocols. Congenital Syphilis. https://cpsp.cps.ca/uploads/studies/ Protocol-congenital-syphilis.pdf

6. Finelli L, Berman SM, Koumans EH, Levine WC. Congenital syphilis. Bull World Health Organ 1998;76 Suppl 2:126-8. PubMed

7. Sheffield JS, Sánchez PJ, Morris G, Maberry M, Zeray F, McIntire DD, Wendel GD Jr. Congenital syphilis after maternal treatment for syphilis during pregnancy. Am J Obstet Gynecol 2002;186(3):569-73. DOI PubMed

8. Provincial Health Services Authority (BC). Interim Guideline on Syphilis Screening in Pregnancy. Vancouver (BC): Perinatal Services BC; September 2019. http://www. perinatalservicesbc.ca/Documents/Guidelines-Standards/ Maternal/Guideline-syphilis-screening-in-pregnancy.pdf

9. Arnold SR, Ford-Jones EL. Congenital syphilis: A guide to diagnosis and management. Paediatr Child Health 2000;5(8):463-9. DOl PubMed

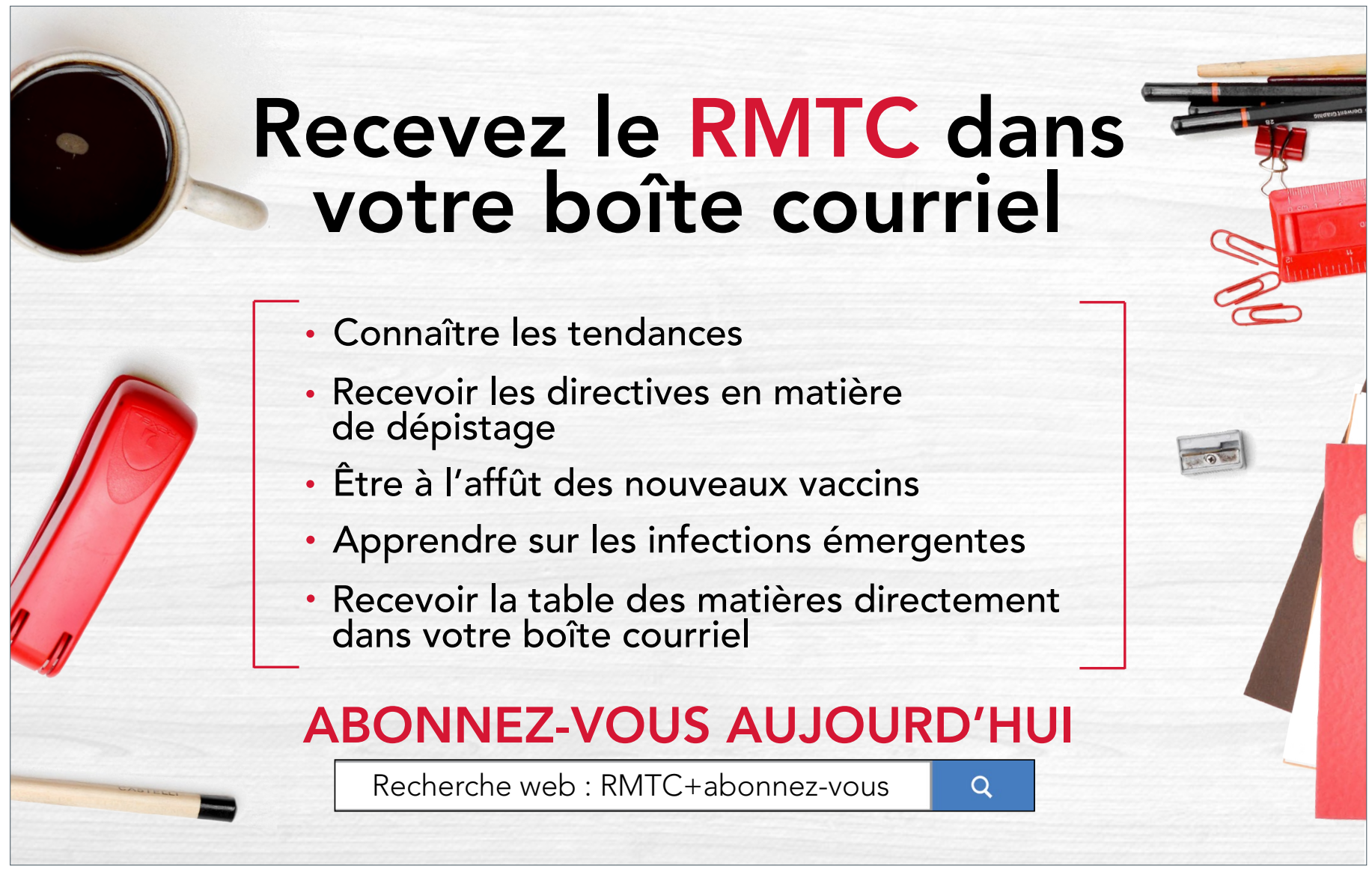

